\title{
Radially isospectral annular membranes
}

\author{
H. P. W. GOTTLIEB
}

School of Science, Griffith University, Nathan, Queensland 4111, Australia

[3 March 2005 ; ]

By utilizing transformations of both the radial coordinate and the radial wave function, densities of annular membranes which are radially isospectral to any given radial density are produced. In particular, new families of annular membrane densities are found which are radially isospectral to a uniform membrane. Some new generalisations for completely isospectral annular membranes (including angle-dependent densities) are also given.

Keywords: isospectral; annular membranes; circular membranes; density; inhomogeneous. 


\section{Introduction}

Axisymmetric vibrations of inhomogeneous annular membranes with radius-dependent densities are often investigated numerically or semi-analytically [Kalotas and Lee (1993), Gutierrez et al (1998), Buchanan and Peddieson (1999), Jabareen and Eisenberger (2001), Subrahmanyam and Sujith (2001), Willatzen (2002)]. It is therefore important to have available some exact solutions for inhomogeneous membranes which may be invoked for checking such computations. Previously, the author (Gottlieb (1992)) showed that an annular membrane with inverse-fourth-power radial density was completely isospectral to a homogeneous membrane, for both Dirichlet (fixed) and Neumann (free) boundary conditions. Furthermore, circular membranes with positive power radial densities were found there to be radially isospectral (i.e. having the same part of the vibration spectrum corresponding to the axisymmetric modes) to circular homogeneous membranes.

The growing importance of the study of isospectrality is reflected for instance by the addition to the recent book by Gladwell (2004), in its second edition, of new chapters on isospectral systems, both discrete and continuous. Discussions of isospectral systems are also included in a new book by Elishakoff (2005). Recently, Knowles and McCarthy (2004) have used some work by Gottlieb (1988) on isospectral circular membranes to establish a connection between isospectral densities and isospectral shapes for vibrating membranes. Isospectral systems may also find applications in the areas of numerical methods, benchmarking, inversion procedures and diagnostics.

In this paper, we investigate more general transformations of the radial membrane wave equation wherein the radial wave function (displacement) may also be modified by 
a radial-dependent multiplicative function, whilst still eventually preserving the radial wave equation form, with modified density distribution. Thereby new families of annular membrane densities are discovered which are radially isospectral to uniform annular membranes. Moreover, for any radial density function, different radially isospectral densities are generated. Some new generalisations for completely isospectral annular membranes (including angle-dependent densities) are also given. 


\section{Formulation}

The radial wave equation for the angle-independent (axisymmetric) modes of a unittension membrane with radial (areal) density function $\phi(\rho)$ is

$$
\frac{\partial^{2} v}{\partial \rho^{2}}+\frac{1}{\rho} \frac{\partial v}{\partial \rho}=\phi(\rho) \frac{\partial^{2} v}{\partial t^{2}}
$$

where $\rho$ is the radial coordinate, $t$ is the time and $v(\rho, t)$ is the transverse displacement. For vibrations with angular frequency $\omega$ this becomes a Helmholtz-type equation (with time-dependence of v suppressed)

$$
\frac{d^{2} v}{d \rho^{2}}+\frac{1}{\rho} \frac{d v}{d \rho}+\omega^{2} \phi(\rho) v=0
$$

A procedure of Gottlieb (2002) for finding isospectral string densities dealt with the ordinary (Cartesian) one-spatial-dimension wave equation. Since (2.1) also only involves one spatial coordinate, albeit of a different type, it is appropriate to apply the same method to this radial equation. Thus the transformations are made to new coordinate and displacement variable

$$
\begin{aligned}
& r=r(\rho), \\
& v(\rho, t)=\gamma(r) u(r, t),
\end{aligned}
$$


with the aim of obtaining a new radial wave equation of the form

$$
\frac{\partial^{2} u}{\partial r^{2}}+\frac{1}{r} \frac{\partial u}{\partial r}=f(r) \frac{\partial^{2} u}{\partial t^{2}}
$$

or, equivalently,

$$
\frac{d^{2} u}{d r^{2}}+\frac{1}{r} \frac{d u}{d r}+\omega^{2} f(r) u=0
$$

of the standard form, corresponding to a radially isospectral membrane (same radial eigenvalues $\omega^{2}$ ) but with different radial density function $\mathrm{f}(\mathrm{r})$.

In (2.3), $\gamma(\mathrm{r})$ is a (positive, non-singular) function of radial coordinate, multiplying the displacement, which allows for more general transformations than just the simple substitution of coordinate with $\gamma=1$. Equation (2.3) preserves the Dirichlet boundary condition of fixed edges $(v=0=u)$.

For brevity, in the following, derivatives with respect to $\mathrm{r}$ will be denoted by a prime (') and derivatives with respect to $\rho$ with a grave (').

After some manipulations are made, the left side of (2.1a) may be expressed as

$$
\gamma\left(r^{\prime}\right)^{2} u^{\prime \prime}+\frac{1}{\rho \gamma}\left(\rho \gamma^{2} r^{\prime}\right)^{\prime} u^{\prime}+\frac{1}{\rho}\left(\rho \gamma^{\prime}\right)^{\prime} u,
$$


whilst the right side of (2.1a) becomes, via (2.3) and (2.4a),

$$
(\gamma \phi / f)\left[u^{\prime \prime}+\frac{1}{r} u^{\prime}\right] .
$$

The expressions (2.5) and (2.6) are therefore required to be equal.

First of all, since $r^{`}=1 / \rho^{\prime}$, equating coefficients of $u^{\prime \prime}$ in (2.5) and (2.6) yields

$$
f(r)=\left(\frac{\partial \rho}{\partial r}\right)^{2} \phi(\rho(r))
$$

(where $\rho(r)$ is the inverse transformation of (2.2)). Equation (2.7) will give the new density function $\mathrm{f}$, radially isospectral to the old density function $\phi$.

Secondly, the coefficient of $u$ in (2.5) must vanish. As expressed in the form in (2.5), this gives simply $\rho \gamma^{\prime}=$ constant, i.e.

$$
\gamma(\mathrm{r}(\rho))=\text { constant } \times \log (\rho)+\text { constant } .
$$

Finally, coefficients of $u^{\prime}$ in (2.5) and (2.6) are equated and (2.7) in the form $\phi / f=$ $\left(r^{\prime}\right)^{2}$ is used. Several further manipulations yield

$$
\left(\rho \gamma^{2}\right)\left(r / r^{\prime}\right)^{\prime}=\left(r / r^{\prime}\right)\left(\rho \gamma^{2}\right)^{\prime}
$$


whence

$$
\log r=\text { constant } \times \int \frac{d \rho}{\rho \gamma^{2}}+\text { constant }
$$

which will lead via (2.8) to $\rho(r)$ and hence via (2.7) to $f(r)$.

Then, by (2.3),

$$
\mathrm{u}(\mathrm{r})=[1 / \gamma(\mathrm{r})] \mathrm{v}(\rho(\mathrm{r}))
$$




\section{Special cases}

In the special case that the first constant appearing in the above result (2.8) is zero, so that $\gamma$ is a constant (which may without loss of generality in (2.3) be taken to be unity), the displacement functions in (2.3) are related by simple coordinate substitution, and both Dirichlet and Neumann (free edge) boundary conditions are preserved. Then the radial coordinate relation $(2.10)$ becomes

$$
\mathrm{r}(\rho)=\text { constant } \times \rho^{\text {constant }},
$$

i.e. there is a power law relation

$$
\rho(\mathrm{r})=\text { constant } \times \mathrm{r}^{\mathrm{p}}
$$

where $\mathrm{p}$ is some real number.

\subsection{Circles}

For circular membranes $0 \leq(\rho, r) \leq b,(3.1 b)$ may be written in the radius-preserving form

$$
\rho(r)=r^{p / b^{p-1}}
$$


Then by (2.7) the density $\phi(\rho)$ is radially isospectral, for both Dirichlet and Neumann boundary conditions, with the density

$$
f(r)=p^{2}\left(\frac{r}{b}\right)^{2 p-2} \phi\left(r^{p} / b^{p-1}\right)
$$

for any $\mathrm{p} \geq 1$ (for circular membrane finite densities). The displacements are related by (2.3):

$$
u(r, t)=v\left(r^{p} / b^{p-1}, t\right)
$$

This recalls the results of an earlier paper (Gottlieb (1992), section 4) for the case of nonconstant radial densities $\phi(\rho)$.

If the parameter in (3.3) is denoted by $\mathrm{p}_{1}$, and a further transformation with parameter $\mathrm{p}_{2}$ is made, it can be shown that the result of these successive transformations is equivalent to a single overall transformation of the same form, with parameter $\mathrm{p}=\mathrm{p}_{1} \mathrm{p}_{2}$. Thus no new type of class is generated by making transformations of transformations. 


\subsection{Annuli}

For annular regions $0<\mathrm{a} \leq(\rho, \mathrm{r}) \leq \mathrm{b}$ (with $\mathrm{a}<\mathrm{b}$ ), the cases $\mathrm{p}<1$ may also be allowed in (3.1b), but the mapped regions are not geometrically congruent, except in the special case $p=-1$. In that case, the radially isospectral systems are related by

$$
\begin{aligned}
& \rho=\frac{a b}{r}, \\
& f(r)=\frac{a^{2} b^{2}}{r^{4}} \phi\left(\frac{a b}{r}\right), \\
& u(r, t)=v\left(\frac{a b}{r}, t\right) .
\end{aligned}
$$

This recalls a result of Gottlieb (1992, section 3), generalising it to non-constant radial densities $\phi(\rho)$.

Moreover, it may be verified by inclusion of angular coordinate terms of the form $\left(1 / r^{2}\right)\left(\partial^{2} u / \partial \theta^{2}\right)$ in the full plane polar coordinate Laplacian that there is actually complete isospectrality for these annuli (i.e. for all modes, including angular modes), as in the result just mentioned, and furthermore this holds even if the densities are generalised to be angle-dependent: $\phi(\rho, \theta)$. This new generalisation states that there is complete isospectrality in the annular case for densities and displacements related by 


$$
\begin{aligned}
& f(r, \theta)=\frac{a^{2} b^{2}}{r^{4}} \phi\left(\frac{a b}{r}, \theta\right), \\
& u(r, \theta ; t)=v\left(\frac{a b}{r}, \theta ; t\right) .
\end{aligned}
$$

This holds for both Dirichlet and Neumann rim conditions. It also holds for mixed boundary conditions (part rim free, part rim fixed as appropriate ) provided that account is taken of (3.5) which shows that inner and outer radii are interchanged in the mapping. A further similar transformation of radial coordinate simply results in the original situation. 


\section{The general transformation}

In the case that a non-constant multiplicative function $\gamma$ of the form (2.8) relates the displacements as appearing in (2.3), radial isospectrality will only be obtained for the Dirichlet boundary condition. Furthermore, because of the logarithmic term in (2.8), only annular regions may be accommodated: $0<\mathrm{a} \leq(\rho, \mathrm{r}) \leq \mathrm{b}$ (with $\mathrm{a}<\mathrm{b}$ ). However, new classes of radial isospectrality are revealed, because of the appearance of a new, free, parameter.

Substitution of (2.8) for $\gamma$ into (2.10) and subsequent integration, and absorption of inessential constants, yield, for $r(\rho)$,

$$
\log \left(\frac{r}{C}\right)=\frac{A}{\log (\rho / B)}
$$

where $\mathrm{A}, \mathrm{B}$ and $\mathrm{C}$ are constants.

The inner and outer radii preserving conditions

$$
\mathrm{r}(\mathrm{a})=\mathrm{a} ; \mathrm{r}(\mathrm{b})=\mathrm{b}
$$

enable 2 constants to be evaluated, and then one eventually obtains

$$
\log \left(\frac{B r}{a b}\right)=\frac{\Lambda(a, b ; B)}{\log (\rho / B)}
$$


where

$$
\Lambda(a, b ; B)=\log (B / a) \log (b / B)
$$

Here, B is some positive constant which leads to a parametrized family of new isospectral densities. In order that the right side of (4.3) does not become singular, we require either $\mathrm{B}<\mathrm{a}$ or $\mathrm{B}>\mathrm{b}$. Thus, in (4.4), $\Lambda<0$.

The inverse transformation $\rho(r)$ can be shown to have the same form as (4.3) with parameter

$$
\overline{\mathrm{B}}=\mathrm{ab} / \mathrm{B} \text {. }
$$

(Note that $\Lambda(a, b ; \bar{B})=\Lambda(a, b ; B)$.) Together with $(2.7)$, since $\left(r^{\prime}\right)^{2}=1 /\left(\rho^{\prime}\right)^{2}$, this confirms true radial isospectrality, i.e. of $\mathrm{f}$ with $\phi$ and of $\phi$ with $\mathrm{f}$.

From (4.3), the radial coordinate transformation here is explicitly

$$
\rho(r)=B \exp \left[\frac{\Lambda(a, b ; B)}{\log (B r / a b)}\right] .
$$

From (2.7) it then follows that annular membranes $0<\mathrm{a} \leq \mathrm{r} \leq \mathrm{b}$ with the densities 


$$
f(r)=B^{2} \Lambda^{2}(a, b ; B) \frac{\exp \left[\frac{2 \Lambda(a, b ; B)}{\log (B r / a b)}\right]}{r^{2} \log ^{4}(B r / a b)} \phi\left(B \exp \left[\frac{\Lambda(a, b ; B)}{\log (B r / a b)}\right]\right),
$$

with $\Lambda(\mathrm{a}, \mathrm{b} ; \mathrm{B})$ given by (4.4), are all radially isospectral, for Dirichlet boundary condition, with the congruent annular membrane $0<a \leq \rho \leq b$ with density $\phi(\rho)$ for each positive $\mathrm{B}<\mathrm{a}$ or $\mathrm{B}>\mathrm{b}$ (and hence with each other).

Significantly, if $\phi$ is constant, (4.7) gives a family of new densities (not obtainable in Gottlieb (1992)) of congruent annular membranes which are all radially isospectral to each other and to that constant density membrane.

The multiplying function $\gamma(\mathrm{r})$ relating the displacement functions in (2.3) is determined via (4.3) and (d/d $\rho)(2.10)$, up to an inessential multiplicative constant, as

$$
\gamma(r)=\frac{1}{\log (\mathrm{Br} / \mathrm{ab})},
$$

so the displacement functions corresponding to (4.7) are related by

$$
u(r)=\log (B r / a b) v\left(B \exp \left[\frac{\Lambda(a, b ; B)}{\log (B r / a b)}\right]\right) .
$$


To determine the result of successive coordinate transformations, it is convenient to index coordinates and to deal with logarithmic quantities. In (4.6), let $\rho=r_{1}$ and $r=r_{2}$ and $\mathrm{s}_{\mathrm{i}}=\log \left(\mathrm{r}_{\mathrm{i}}\right)$. Let $\beta_{1}=\log \left(\mathrm{B}_{1}\right), \alpha=\log (\mathrm{ab})$, and $\Lambda_{1}$ involve $\mathrm{B}_{1}$ according to (4.4). Then one finds

$$
s_{1}\left(s_{2}\right)=\frac{\beta_{1} s_{2}+\left[\Lambda_{1}+\beta_{1}\left(\beta_{1}-\alpha\right)\right]}{s_{2}+\left(\beta_{1}-\alpha\right)},
$$

which is a real bilinear transformation. Now replace 1 by 2 and 2 by 3 in (4.10) to obtain the corresponding expression for a subsequent transformation $\mathrm{s}_{2}\left(\mathrm{~s}_{3}\right)$ with parameter $\mathrm{B}_{2}$. Set $\Gamma=[\log (a)][\log (b)]$. Then, for $i=1,2$,

$$
\Lambda_{\mathrm{i}} \equiv \log \left(\mathrm{B}_{\mathrm{i}} / \mathrm{a}\right) \log \left(\mathrm{b} / \mathrm{B}_{\mathrm{i}}\right)=-\beta_{\mathrm{i}}^{2}+\alpha \beta_{\mathrm{i}}-\Gamma \quad .
$$

For the result of these two successive transformations, a certain amount of algebra leads to the result that $s_{1}\left(s_{2}\left(s_{3}\right)\right)$ is expressible in exactly the same form as (4.10), i.e. $s_{1}\left(s_{3}\right)$, with overall transformation parameter $\Lambda_{3}=\Lambda_{1,2}$ given by

$$
\Lambda_{1,2}=-\beta_{1,2}^{2}+\alpha \beta_{1,2}-\Gamma
$$

where explicitly 


$$
\beta_{1,2}=\frac{\beta_{1} \beta_{2}-\Gamma}{\beta_{1}+\beta_{2}-\alpha}
$$

so that correspondingly

$$
\mathrm{B}_{1,2}=\exp \left[\frac{\left(\log \mathrm{B}_{1}\right)\left(\log \mathrm{B}_{2}\right)-(\log \mathrm{a})(\log \mathrm{b})}{\log \left(\mathrm{B}_{1} \mathrm{~B}_{2}\right)-\log (\mathrm{ab})}\right] .
$$

That is to say, it has now been demonstrated that a transformation of a transformation (with $\mathrm{B}_{2} \neq \overline{\mathrm{B}}_{1}$, for finiteness) does not produce a new class of membrane, but simply another radially isospectral annular membrane within the same class, just with a different value of the parameter B given explicitly in terms of the intermediate transformation parameters by (4.14). 


\subsection{Interchange of rim radii}

It was noted in sub-section 3.2 above (c.f. Gottlieb (1992, section 3)) that it is possible to retain the geometric congruency of annular regions $0<a \leq(\rho, r) \leq b$ whilst interchanging the mappings of inner and outer radii. Thus (4.2) may also be replaced by

$$
\mathrm{r}(\mathrm{a})=\mathrm{b} ; \mathrm{r}(\mathrm{b})=\mathrm{a} .
$$

Then (4.1), and a suitable redefinition of constants so that the argument of the logarithmic terms conforms with that in (4.6-7), eventually lead to the radial coordinate transformation

$$
\rho_{l}(r)=\frac{a b}{B} \exp \left[\frac{-\Lambda(a, b ; B)}{\log (B r / a b)}\right]
$$

for any positive constant $\mathrm{B}<\mathrm{a}$ or $\mathrm{B}>\mathrm{b}$.

The further set of densities $f_{I}(r)$ radially isospectral with density $\phi(\rho)$ are correspondingly given by

$$
f_{l}(r)=\frac{a^{2} b^{2}}{B^{2}} \Lambda^{2}(a, b ; B) \frac{\exp \left[\frac{-2 \Lambda(a, b ; B)}{\log (B r / a b)}\right]}{r^{2} \log ^{4}(B r / a b)} \phi\left(\frac{a b}{B} \exp \left[\frac{-\Lambda(a, b ; B)}{\log (B r / a b)}\right]\right)
$$


for all such B as above. Comparison shows that this second set (4.17) is distinct from the set (4.7). 


\section{Conclusion}

In this paper, new classes of isospectral and radially isospectral annular membranes have been discovered. The work significantly extends the classes of membrane densities possessing known complete or radial spectra. As well as this intrinsic interest, these formulae may also be used for checking various numerical approaches when applied to inhomogeneous membranes, given isospectrality to other and simpler membranes with known spectra. 


\section{REFERENCES}

BUCHANAN, G.R. \& PEDDIESON, J. (1999) Vibration of circular, annular membranes with variable density. J. Sound Vib. 226, 379-382.

ELISHAKOFF, I. (2005) Eigenvalues of Inhomogeneous Structures: Unusual closed-form solutions. Boca Raton: CRC Press.

GLADWELL, G. M. L. (2004) Inverse Problems in Vibration, 2nd edition. Dordrecht: Kluwer.

GOTTLIEB, H. P. W. (1988) Density distribution for isospectral circular membranes. SIAM J. Appl. Math. 48, 948-951.

GOTTLIEB, H. P. W. (1992) Axisymmetric isospectral annular plates and membranes. IMA J. Appl. Math. 49, 185-192.

GOTTLIEB, H. P. W. (2002) Isospectral strings. Inverse Problems 18, 971-978.

GUTIERREZ, R. H., LAURA, P. A. A., BAMBIL, D. V., JEDERLINC, V. A. \& HODGES, D.H. (1998) Axisymmetric vibrations of solid circular and annular membranes with continuously varying density. J. Sound Vib. 212, 611-622.

JABAREEN, M. \& EISENBERGER, M. (2001) Free vibrations of non-homogeneous circular and annular membranes. J. Sound Vib. 240, 409-429.

KALOTAS, T.M. \& LEE, A.R. (1993) The modes of a circular membrane of non-uniform radial density. Acustica 78, 220-225.

KNOWLES, I. W. \& McCARTHY, M. L. (2004) Isospectral membranes: a connection 
between shape and density. J. Phys. A: Math. Gen. 37, 8103-8109.

SUBRAHMANYAM, P.B. \& SUJITH, R. I. (2001) Exact solutions for axisymmetric vibrations of solid circular and annular membranes with continuously varying density. J. Sound Vib. 248, 371-378.

WILLATZEN, M. (2002) Exact power series solutions for axisymmetric vibrations of circular and annular membranes with continuously varying density in the general case. J. Sound Vib. 258, 981-986. 